\title{
DEVELOPMENT AND TESTING OF AN ENERGY HARVESTING TILE
}

\author{
Ganesh Sevagamoorthy ${ }^{1}$ and Jayabal $\mathrm{K}^{2}$ \\ ${ }^{1}$ Indian Institute of Information Technology Design and Manufacturing, Kancheepuram, \\ India. \\ ${ }^{2}$ Indian Institute of Information Technology Design and Manufacturing, Kancheepuram, \\ India.
}

\begin{abstract}
This paper presents development and experimental analysis of a piezoelectric mounted flexible beam attached with an oscillating tile that can be used to scavenge energy from footsteps in crowded places. The energy harvesting system consists of a piezoelectric bimorph cantilever beam inside a hollow box which connects to the ground with the help of springs. Multiple piezoelectric patches are pasted on the beam. When the foot is placed on the tile and removed, the hollow box displaces in turn causing the beam to oscillate harvesting electrical power. A prototype is developed and the performance of the piezoelectric energy scavenging tile is tested. The variations of power output for different load resistances are obtained and the optimal load resistance is suggested.
\end{abstract}

\section{KEYWORDS}

Energy harvesting, Piezoelectric material, Bi-morph cantilever beam.

\section{INTRODUCTION}

The world energy production sector is in transition and is nowadays called to face great challenges in a context in which the fossil fuel reserves are running out, while the energy demand steadily increases. On the other hand, the rising cost and the related environmental issues make the use of conventional energy resources more and more difficult [1]. There are an estimated 1.3 trillion barrels of proven oil reserve left in the world's major fields, which at present rates of consumption will be sufficient to last 40 years. By 2040, production levels may be down to 15 million barrels per day - around $20 \%$ of what we currently consume. It is likely by then that the world's population will be twice as large, and more of it industrialised and therefore oil dependent [2]. Energy harvesting has been of interest to many researchers for decades. Researchers have put much effort into energy conversion and it is recently suggested that piezoelectric materials are one of the best options in energy conversion from mechanical into electrical [3]. Piezoelectric materials produce electrical charges when subjected to dynamic strain. These materials can be used to capture and store vibrational energy. The Energy storage characteristics of Piezo-generator and the effect of the capacitance of the capacitor and the initial voltage are discussed by Umeda et al [4]. The various piezoelectric devices available have been discussed in detail by Sodano et al [5]. There are two methods to accumulate the charge produced by the piezoelectric harvester. The first one uses capacitors and the second one nickel metal hydride batteries. The rechargeable batteries are found to have more desirable qualities than the capacitor [6]. 
A lot of energy is being spent by pedestrian traffic while walking in crowded places like malls, train stations, airports, etc. Here, energy expended when the foot is placed on the ground results in vibration which can be converted into electrical energy. This can be done, among others, with the help of multiple piezoelectric patches placed on either side of the cantilever beam. The energy from the footstep can be transferred to the beam through a box. An improved design based on aforementioned idea was discussed and a numerical analysis was also reported in a recent work [7]. The main idea of the present work is to design a simple and cheap energy harvesting device to scavenge the energy expended while some one walks over the energy scavenging tile.

\section{DESIGN}

The design of the energy scavenging tile consists of a box which is placed in a cavity connected with the base through springs. This box has a cantilever beam inside and several piezoelectric patches are pasted on either side of the beam as shown in Figure 1. As it can be seen, when the foot is placed on the box, the box displaces down and as the person removes his or her foot, it comes back to its original position due to spring action. Inertia causes the beam within the box to vibrate thereby producing electric power. Figure 2 displays the schematic sketch of the energy harvesting tile and various parts.

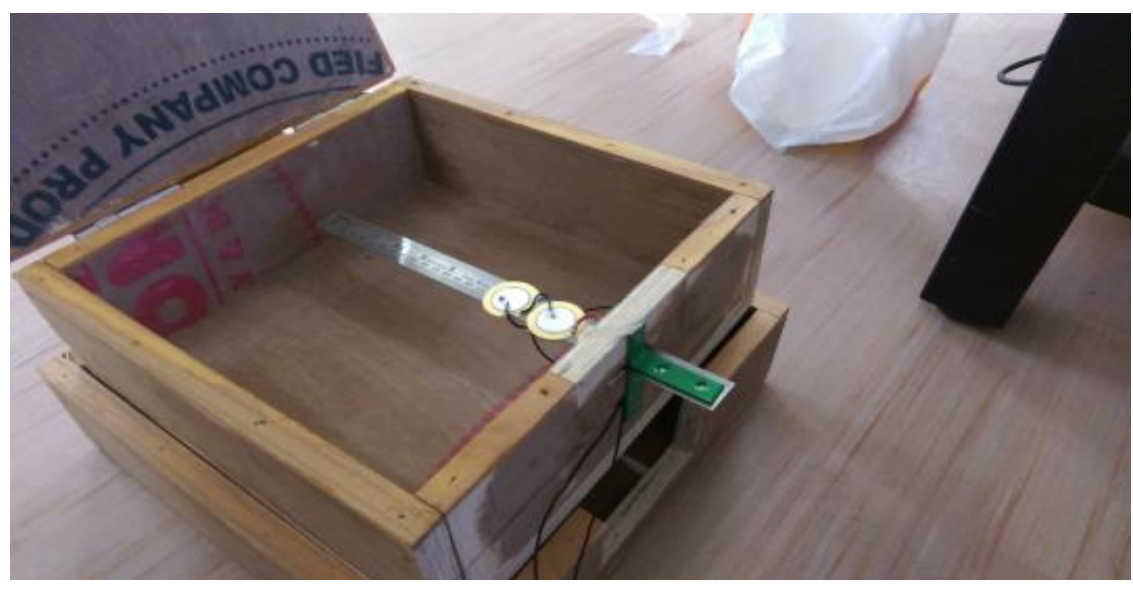

Figure 1. Energy scavenging tile with the piezo mounted beam

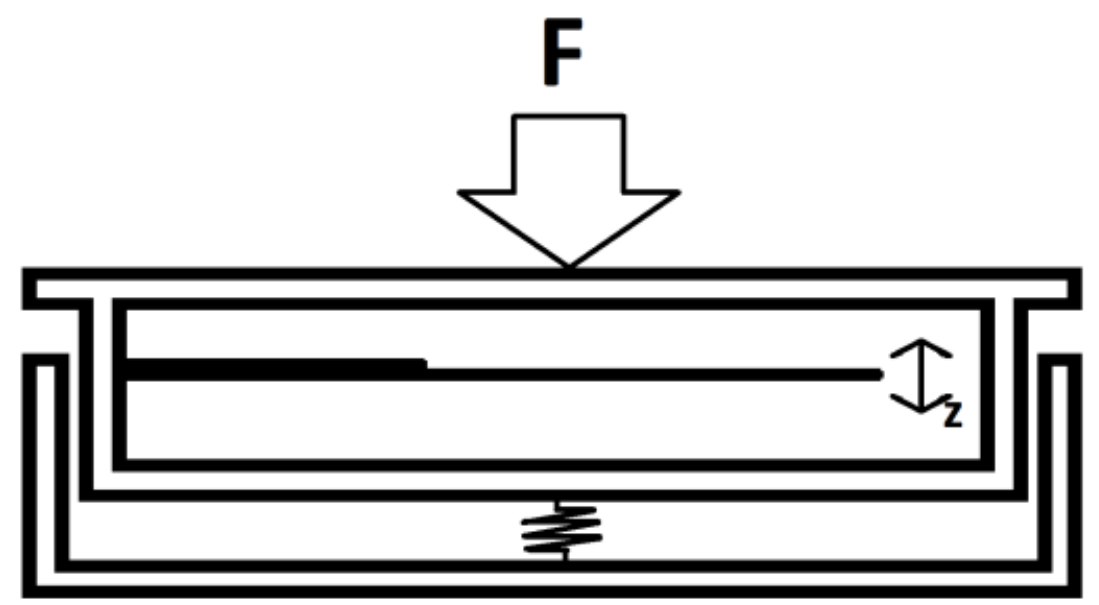

Figure 2. Schematic view of the Energy harvesting tile and its working 
The prototype consists of a wooden box and a wooden platform with inner dimensions $34 \mathrm{~cm} \mathrm{x}$ $34 \mathrm{~cm} \times 10 \mathrm{~cm}$. The box can displace up and down on the platform as it is supported by eight compression springs. One side of the box has a slot to accommodate the beam. Two L-shaped hinges are attached on either side of the slot to tightly hold the beam in place. The inside box has outer dimensions of $33.5 \mathrm{~cm} \times 33.5 \mathrm{~cm} \times 10 \mathrm{~cm}$. The cantilever beam is a steel ruler of length $27.5 \mathrm{~cm}$, made of stainless steel and has six piezoelectric patches pasted on it (three on either side of the beam) and these piezoelectric patches are connected in series. The piezoelectric material used is a piezo buzzer element, each having a radius of $2.5 \mathrm{~cm}$. Due to the restriction arising out of the comfort of pedestrian, the vertical displacement of the box is constrained to $1 \mathrm{~cm}$.

\section{EXPERIMENT}

The output from the piezoelectric element is connected onto a breadboard across a load resistance. The voltage across the load resistance is measured and plotted with the help of an oscilloscope. For characterizing the prototype, a force of $200 \mathrm{~N}$ is applied on the top of the hollow box so it has a deflection of $10 \mathrm{~mm}$ and resulting output voltage is plotted on the oscilloscope. The system described here is designed to utilize the $z$-axis vibration as the only vibration source for the device. The output voltage is measured for various values of load resistances. The complete experimental setup with the oscillating box and necessary measuring devices is shown in Figure 3.

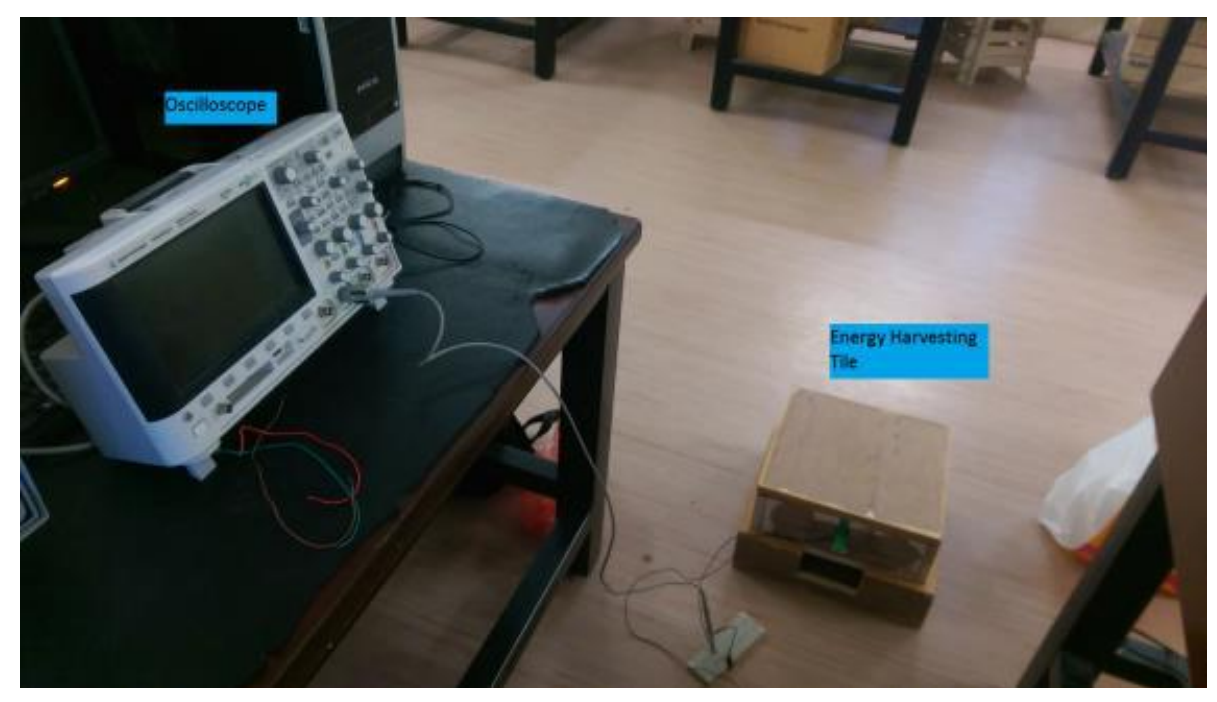

Figure 3. Experimental Setup

\section{RESULTS AND DiSCUSSION}

The energy scavenging was subjected to a constant transverse load and its power output was measured with the help of an oscilloscope. The values of voltage obtained are used to find the power developed by the device and check the performance of the energy harvesting device. Two cases have been studied with an open circuit and with a load resistance.

\subsection{Open Circuit}

The output voltage obtained from the experiment performed on the energy scavenging tile are shown in the Figure 4. The experimental results show that the tile produces a peak AC Voltage of 56.8 Volts in the open circuit condition. 


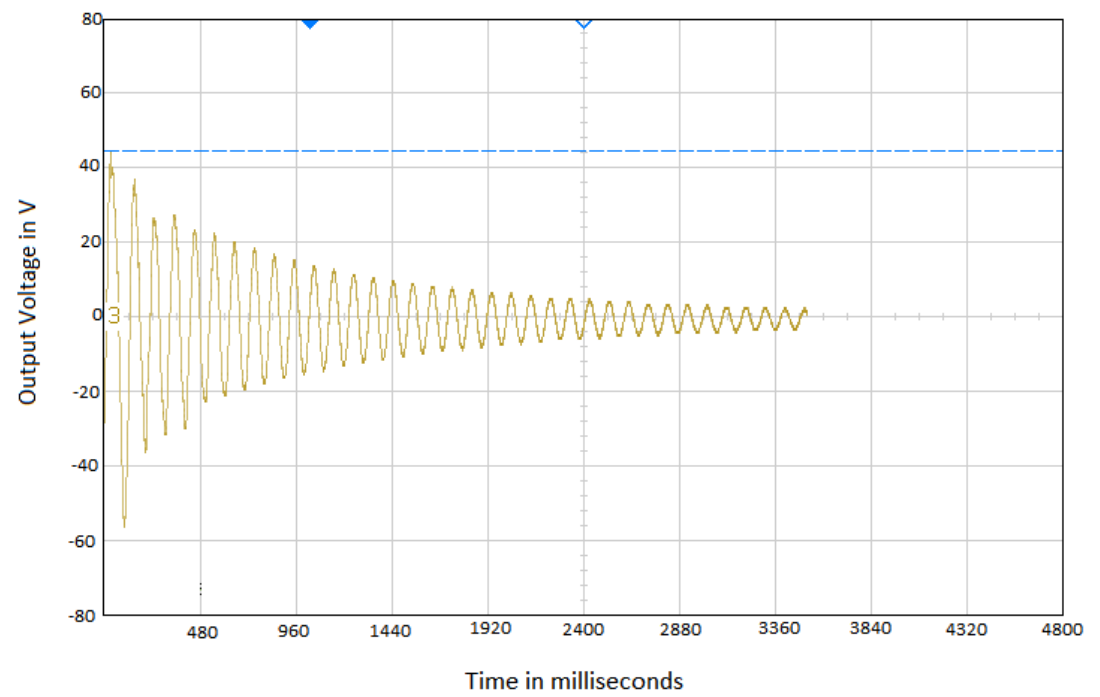

Figure 4. Output voltage for open circuit

\subsection{Circuit with resistive load}

The voltage across the circuit has been measured for eleven different resistances. The output voltage waveform for $180 \mathrm{kilo}$ ohm resistance is shown in Figure 5. It has a peak voltage of 4.7 volts. The output voltages for different resistances has been plotted in Figure 6. It can be seen that the output voltages increase with load resistances. In Figure 7, a plot between the current and load resistances shows that the current decreases with increase in load resistance. The piezoelectric energy harvester produces a max power of around $122.72 \mu \mathrm{W}$ at a load resistance near to 180 kilo ohms. This is shown in a graph between the max power output and load resistance in Figure 8.

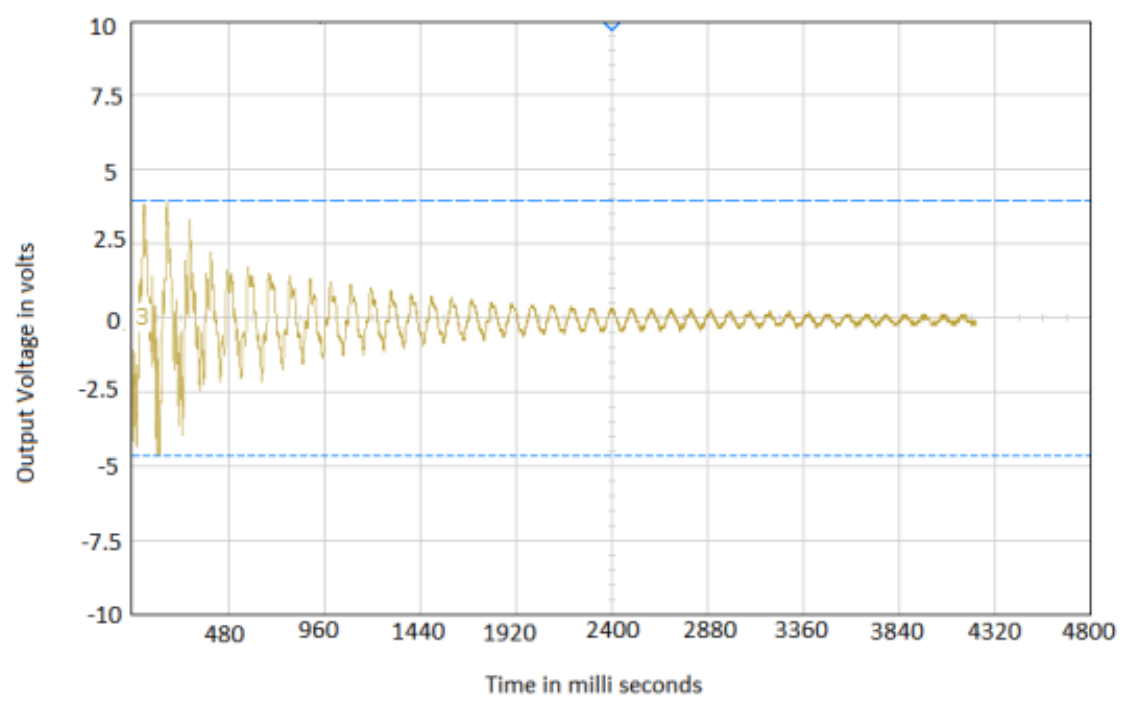

Figure 5. Output voltage for 180 kilo-ohms load resistance 
International Journal on Cybernetics \& Informatics (IJCI) Vol. 5, No. 1, February 2016

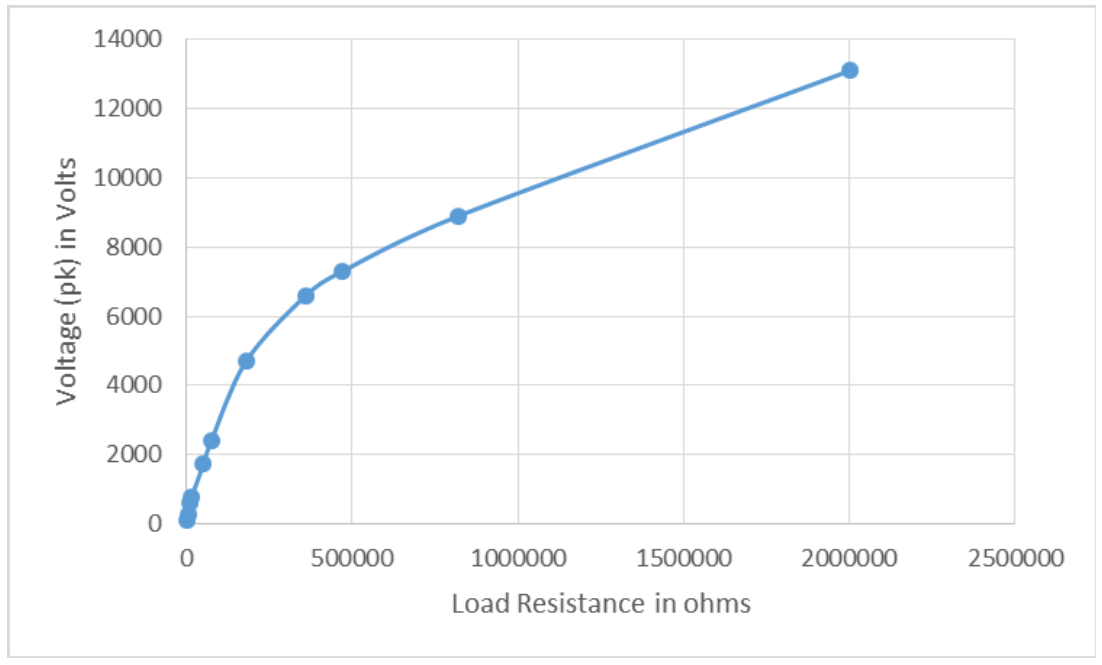

Figure 6. Voltage peak vs Load resistance

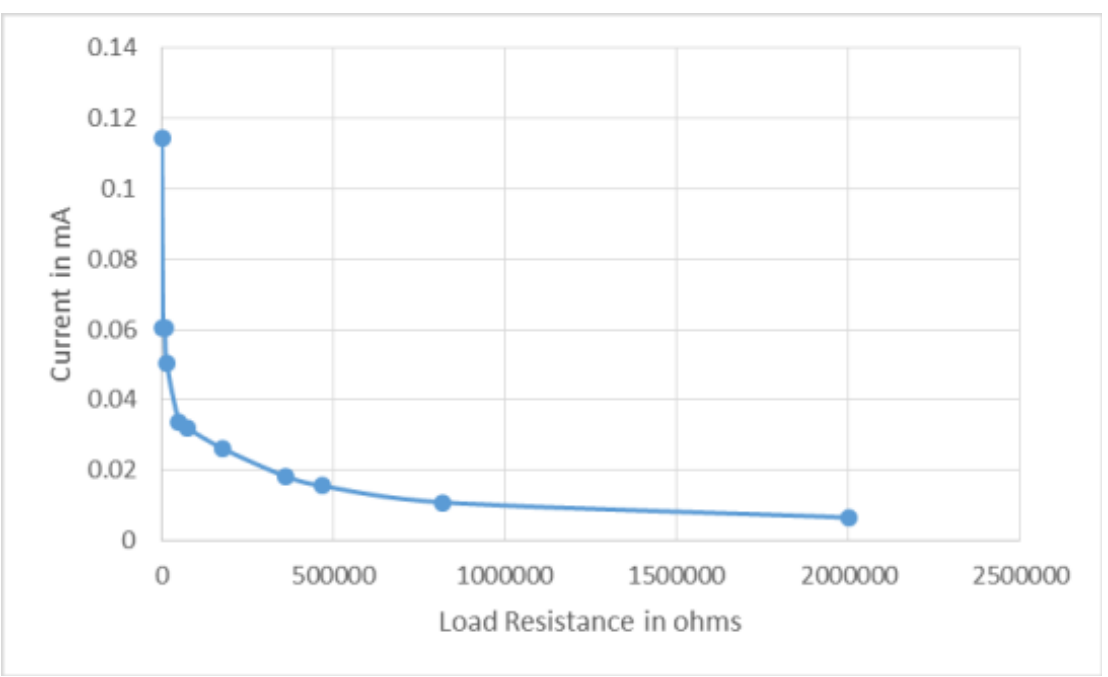

Figure 7. Current peak vs Load resistance

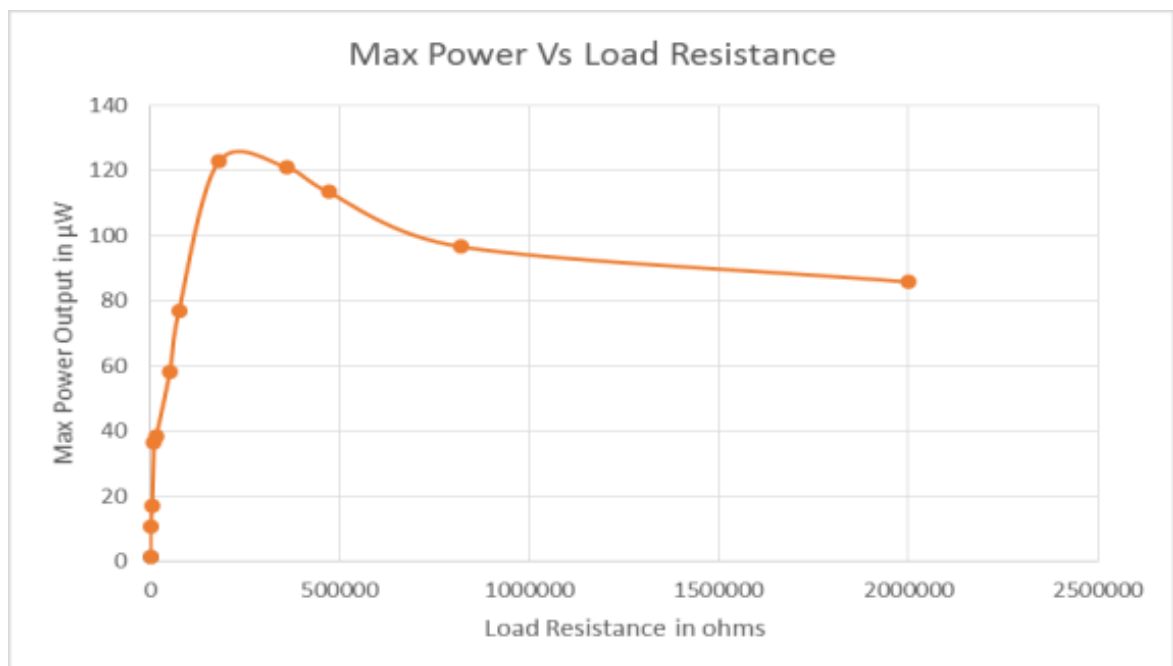

Figure 8. Max power output vs Load resistance 


\section{CONCLuSiON}

In this work, it is attempted to develop a simplified energy harvesting tile and its performance was tested for different load resistances. It is found that a force of 20 kilo-grams resulted in producing a peak AC power of around 122 micro-Watts. This design can further be extended with more beams placed inside the hollow box to produce more power which can be harvested while someone walking on the tile. In addition, the platform base may be pasted with piezo stacks, as is conventionally done, which will enhance the power output. This tile can also be used to get statistical data in shops and also for security system as a part of an intruder detecting system owing to its ability to detect small change in pressure.

\section{REFERENCES}

[1] A. Messineo, G. Panno (2011) "LNG cold energy use in agro-food industry: a case study in Sicily", Journal of Natural Gas Science and Engineering, 3: 356-363.

[2] http://www.imeche.org.

[3] S.R. Anton, H.A. Sodano (2007) "A review of power harvesting using piezoelectric materials (20032006)", Smart Materials \& Structures, 16:R1-R21.

[4] M. Umeda, K. Nakamura, S. Ueha (1997) "Energy Storage Characteristics of a Piezo-Generator Using Impact Induced Vibration." Japanese Journal of Applied Physics, Vol. 35, Part 1, No. 5B, pp. 3146-3151.

[5] H.A. Sodano, G. Park, D.J. Inman (2005) "Comparison of Piezoelectric Energy Harvesting Devices for Recharging Batteries", Journal of Intelligent Material Systems and Structures, 16(10), 799-807.

[6] H.A. Sodano, G. Park, D.J. Inman (2005) "Generation and Storage of Electricity from Power Harvesting Devices", Journal of Intelligent Material Systems and Structures, 16(1), 67-75.

[7] A. Messineo (2012) "Piezoelectric bender transducers for energy harvesting applications", Energy Procedia $14.39-44$. 